\title{
PERFIL POTENCIAL DE INADIMPLÊNCIA NO USO DO CARTÃO DE CRÉDITO: ANALISE DE TÉCNICA DE CLUSTERS
}

Leonardo Jantsch (Universidade do Vale do Rio dos Sinos - Unisinos, Rio Grande do Sul, Brasil) - ljantsch@gmail.com

Adolfo Alberto Vanti (Universidade do Vale do Rio dos Sinos - Unisinos, Rio Grande do Sul, Brasil) - Avanti@unisinos.br

Angel Cobo (Universidad de Cantabria, Cantabria, Espanha) - angel.cobo@unican.es

Rocio Rocha (Universidad de Cantabria, Cantabria, Espanha) - eliana.rocha@unican.es

\section{RESUMO}

O objetivo deste trabalho está relacionado com a identificação de perfil potencial de inadimplência no uso do cartão de crédito, priorizando a análise com método de clusterização de garimpagem de dados. Para atingir o propósito do trabalho se utilizou a metodologia CRISP-DM como contextualização e logo se especificou as etapas de preparação de dados, modelagem e algumas análises utilizando-se Excel e WEKA. O contexto de aplicação é a carteira de uma instituição financeira emissora de cartões com atuação em onze estados brasileiros, sendo que os dados trabalhados correspondem aos saldos mensais de janeiro/13 a dezembro/14 da carteira anônima de cartão crédito dos portadores adimplentes e inadimplentes integrados ao perfil de cada cliente. A partir dos resultados encontrados conclui-se que dados de perfil como idade do portador, por exemplo, indicam tendência de comportamento que pode ser utilizado na gestão da carteira e como foco de ação na orientação de usuários de cartão.

Palavras-Chave: Cartão de Crédito; Adimplência, Inadimplência; Data Mining; Clusters.

\section{PROFILE POTENTIAL INSOLVENCY IN CREDIT CARD USE: TECHNICAL ANALYSIS OF CLUSTERS}

\begin{abstract}
The objective of this paper is related to the identification of potential insolvency profile in the use of credit card, prioritizing the analysis with clustering method of data mining. To achieve the aim was used the CRISP-DM methodology as contextualization and soon specified data preparation steps, some modeling and analysis using Excel and WEKA. The application context is the portfolio of a financial institution issuing cards with operations in eleven Brazilian states, and the data worked correspond to monthly balances January/13 to December/14 the anonymous credit card portfolio of solvency and insolvency consumers integrated the profile of each customer. From the results it is concluded that profile data such as age of the consumer, for example, indicate pattern of behavior that can be used in portfolio management and focus action on the orientation of card users.
\end{abstract}

Keywords: Credit Card; Solvency, Insolvency; Data Mining; Clusters. 


\section{INTRODUÇÃO}

O crédito de cartão de crédito é cada vez mais representativo no cenário brasileiro e a carteira de cartões de crédito em junho/2014 alcançou $12,1 \%$ do total de crédito disponível a pessoas físicas (BACEN a, 2014). No primeiro semestre, o cartão de crédito apresentou crescimento da ordem de 14\% (BACEN c, 2014), e estima-se, com o cenário de alta de juros, que este desempenho se mantenha para os próximos períodos, mas com um crescimento mais acentuado do crédito a vista em detrimento do crédito rotativo.

Entre fevereiro e junho de 2014 a inadimplência do cartão aumentou de 6,4\% para $6,7 \%$, e a operação com maior risco de inadimplência, o crédito rotativo em junho/2014 era de R \$ 29,4 bilhões, representando $20 \%$ da carteira de crédito total do cartão. Considerando que o ano de 2015 o Brasil prevê crescimento mínimo ou nulo do PIB com retração de diversos mercados, juros e impostos mais altos mas com uma cultura de gastos que ainda pode demorar para mudar/diminuir, prevê-se um aumento de inadimplência que pode alcançar percentuais além dos patamares atuais.

Considerando o contexto econômico e comportamento de gastos do consumidor brasileiro por vezes com baixo controle, este trabalho objetivou analisar o perfil potencial de inadimplência no uso do cartão de crédito sob a técnica de clusters de garimpagem de dados. Com isso a questão de pesquisa foi definida em: Qual o perfil potencial de inadimplência no uso do cartão de crédito? Essa questão de pesquisa está posicionada com sua solução utilizando-se foco na técnica de clusters para garimpagem de dados como sequência de trabalhos científicos aplicados à realidade empresarial.

Integrando o enfoque teórico e prático também posiciona-se este trabalho no contexto dos enfoque de Oh, Johnston (2014); Mukherjee, Kawde (2014); e Bendle, Horne (2014), os quais descrevem o modelo global da indústria de cartões e as principais características dos consumidores que optam por esta modalidade de pagamento. Com isso, as instituições financeiras podem se preparar ainda melhor para mitigar riscos do uso excessivo desse tipo de modalidade de crédito, protegendo seus ativos mas também protegendo o próprio cidadão de endividamentos pouco controláveis.

\section{REVISÃO DA LITERATURA}

\subsection{Inadimplência do Cartão de Crédito}

Para Rochet e Tirole (2003), o mercado de dois lados da indústria de cartões está estruturado em uma plataforma de duas, três ou quatro partes, sendo que a diferença entre as estruturas está na figura da instituição financeira emissora, normalmente bancos, e do credenciador da rede de captura de transações.

O modelo mais complexo constitui-se de quatro partes, conforme Akers et al. (2005), apresentando uma bandeira com muitos titulares de cartões, estabelecimentos e credenciadores de várias instituições financeiras. Neste modelo, a bandeira desempenha um papel importante de imposição de regras para a emissão de cartões, compensação e liquidação financeira de transações, publicidade e promoção da marca, autorização de transações, avaliação de taxas e alocação de receitas entre os participantes da transação. As bandeiras Visa@ e MasterCard@ seguem esse modelo.

Com exceção ao modelo de países desenvolvidos, no Brasil os emissores de cartão de crédito no Brasil não assumem o custo do dinheiro no tempo pelo descasamento de prazos entre a liquidação da fatura e o pagamento aos estabelecimentos. Isto ocorre no nosso mercado pois os portadores pagam sua fatura em média vinte oito dias após a 
compra e o estabelecimento recebe em média trinta dias após a compra (BACEN b, 2010, pg.7).

Na visão do portador do cartão no modelo descrito, encontram-se três principais tipos de produtos (BENDLE, HORN, 2014), o Cartão de débito, o Cartão de crédito e o Cartão pré-pago, todos eles de operação bem conhecida do público em geral.

Cabe analisar com maior especificidade que o cartão de crédito permite o pagamento das compras após um período sem juros, em geral, de até 45 dias. No final deste período, o consumidor pode optar por pagar o montante total em dívida, caso em que não haverá juros acumulados. Como alternativa, o consumidor pode optar por pagar apenas uma parte da dívida, sendo a parcela restante denominada de "rotativo", saldo devedor este que passa a sofrer a incidência de juros acumulados sobre o montante utilizado, com todas as taxas e juros pagos para o emissor do cartão (MUKHERJEE, KAWDE, 2014).

As taxas de inadimplência do portfólio atual de empréstimos não afetam o lucro do período, mas terão um efeito negativo sobre os resultados futuros por se tornarem perdas de crédito (OH, JOHNSTON, 2014). Conforme Santos e Famá (2007) as carteiras de créditos rotativos (cheque especial e cartão de crédito) com elevadas taxas de juros cobradas de clientes captados, sofrem considerável impacto em situações adversas.

Essas situações adversas se relacionam a perda total ou parcial da renda salarial do portador e de eventos sistêmicos que promovam a elevação das taxas de juros, forçando à renegociação com redução do spread e da lucratividade. Adicionalmente segundo Getter (2008) o crédito rotativo possui o maior risco de inadimplência entre as formas de empréstimos bancários, elevando os custos operacionais.

Os aspectos comportamentais também influenciam nos níveis de demanda e composição das carteiras como o analisado em Shefrin e Nicols (2014) em que o comportamento de usuários de cartão de crédito alcança $40 \%$ dos usuários atribuindo grande importância para o controle de suas finanças mas com $44 \%$ desse grupo relatando baixa confiança na capacidade de gerir as suas finanças usando por exemplo a tecnologia online.

Na concessão do crédito ao consumidor são encontrados três estágios, conforme Oh e Johnston (2014): aplicação e triagem; aprovação de crédito e pagamentos; inibição e perda. Estas três etapas compreendem o ciclo de crédito na gestão de risco. Uma ênfase maior ocorre na literatura com o processo de avaliação de crédito da uma nova conta, mas há pouca abordagem com o uso de modelos de análise no gerenciamento contínuo das contas que formam a carteira. (COFFMAN e CHANDLER, 1983; OH e JOHNSTON, 2014). Mesmo utilizando-se de diversas técnicas de controle de inadimplência, ainda assim o credor enfrenta dificuldades em prever os clientes que são mais propensos a gerarem as perdas por inadimplência.

SHIH et al. (2011) concluíram que através da aplicação de técnicas de mineração de dados é possível decifrar o comportamento dos clientes e análise dos riscos. Utilizando um conjunto de regras de agrupamento foi possível identificar com antecedência grupos de clientes de alto risco, possibilitando negar o pedido de cartão. Para aqueles que já eram portadores de cartões, pôde-se iniciar a redução do crédito disponível e aumentar os pagamentos mínimos para obter recurso financeiro quanto possível antes que se tornassem inadimplentes. Nesse sentido é que esse trabalho estrutura um posicionamento metodológico e avança na técnica de agrupamento/clusterizização, utilizando-se do software Weka e de rotinas complementares que gerarão esse maior controle deste tipo de operação financeira. 


\subsection{Data mining (DM)}

Data mining (DM) é um processo de descoberta de conhecimento em banco de dados com o uso de estatística, matemática, inteligência artificial e aprendizagem automática, o qual extrai e identifica informações de banco de dados para realizar previsões de específicos cenários (TURBAN et al., 2009) ou padrões e regras significativas BERRY e LINOFF (1997). Também define estratégias para tratamento de significativos volumes de informação identificando erros e não conformidades de dados com as operações corporativas (WITTEN e FRANK, 2005).

Silva et al. (2013) analisaram aplicações no setor de pagamentos eletrônicos via cartões de crédito com o uso de mineração de dados relacionando clientes e pagamentos. Também Yeh e Lien (2009, p. 2473) concluem ser possível avaliar casos de inadimplência em clientes de cartão de crédito.

As informações encontradas seguem, conforme Sharma, Osei-Bryson e Kasper (2012), etapas como o entendimento sobre negócio, preparação dos dados, modelagem, avaliação e implantação. A estruturação dessas etapas gera um ciclo de melhoria de conformidade empresarial, governança tecnológica e principalmente como é apresentado nesse trabalho, uma sistematização para a mitigação de riscos. Logicamente que as etapas de DM podem receber adaptações em função do modelo de negócio e da própria necessidade gerada, bem como da flexibilidade de técnica utilizada (ROCHA, COBO, VANTI, 2013).

As tarefas de DM podem ser divididas em duas categorias, as Preditivas e as Descritivas (TAN, STEINBACH e KUMAR, 2005), sendo que as primeiras tratam de antecipar informação (SHMUELI, KOPPIUS, 2011), enquanto que as descritivas buscam derivar padrões através de tendências, grupos e correlações para consolidar relações entre os dados relacionados a situações de risco (BAJO et al., 2012). Esse trabalho posiciona-se na consolidação de relações descritivas, direcionando enfoque à técnica de clusterização e complementado com análise em planilha Excel@.

\section{PROCEDIMENTOS METODOLÓGICOS}

Os algoritmos de DM para aplicação à pesquisa foram selecionados com base em Turban (2009, pp. 155-156), optando-se pela categoria Agrupamento/Cluster para em função da distinção do Perfil dos Clientes que utilizam cartões de crédito. A Classificação analisa dados históricos armazenados em um banco de dados e gera automaticamente um modelo que possa prever o comportamento futuro. A Árvore de decisão está contextualizada na classificação de um número finito de classes com base nos valores de variáveis de entrada e considera uma hierarquia de declarações se-então. A Associação ocorre com as relações entre itens que ocorrem juntos em determinado registro. $\mathrm{O}$ Agrupamento ou cluster divide o banco de dados em segmentos que compartilham qualidades semelhantes e que inicialmente não são conhecidos.

Este trabalho desenvolveu a categoria de agrupamento ou clusterização para aplicação em cartões de crédito, integrando-se também com uma análise descritiva realizada em Excel@. Em trabalho futuro a técnica de Associação será utilizada na sequencia deste mesmo trabalho. Também pode-se relacionar com o processamento CRISP-DM em que aprofundou-se a etapa de entendimento do negócio e na evidenciação da formação da carteira de cartões e a sua evolução até a caracterização da inadimplência JANTSCH, COSER, LAZZARI, VANTI (2014). 


\section{CONTEXTO DA PESQUISA}

O contexto da pesquisa é uma instituição financeira com atuação em onze estados brasileiros, sendo eles: Rio Grande do Sul, Santa Catarina, Paraná, São Paulo, Mato Grosso do Sul, Mato Grosso, Tocantins, Pará, Rondônia, Goiás e Rio de Janeiro. É emissora de uma bandeira própria, que representam $11 \%$ de suas operações, e também atua na emissão de cartões Visa $@$, que representam $89 \%$ das operações. Em agosto/2014 o volume de cartões emitidos era de 2,1 milhões, sendo que deste total, $24,7 \%$ são cartões ativos. A carteira total de crédito neste período representava aproximadamente $\mathrm{R} \$ 550$ milhões. Esse trabalho centrou-se em dados de 3 regionais, selecionadas por apresentarem o maior volume de cartões com saldos em carteira.

Na continuação é apresentada a seção com o ciclo metodológico de clusterização, sendo que uma primeira etapa é a de análise em gerador de SAD em Excel@ para evidenciar e avaliar os dados obtidos. Ressalta-se aqui que os dados dos clientes foram tratados de maneira anônima.

\section{IDENTIFICAÇÃO DAS ETAPAS DA GARIMPAGEM DE DADOS}

Para tratamento dos dados inicialmente foi-se definindo o entendimento do negócio das operações de cartões de crédito da instituição emissora dos mesmos conforme DM (JANTSCH, COSER, LAZZARI, VANTI, 2014, pág. 7):

a) A etapa inicial ocorre quando portador do cartão de crédito opta por efetuar o pagamento com seu cartão ao adquirir um bem ou serviço;

b) $\mathrm{O}$ estabelecimento transmite para o credenciador os dados do cartão e o montante da transação;

c) $\mathrm{O}$ credenciador encaminha esta informação à instituição financeira emissora do cartão, solicitando autorização para a transação;

d) $\mathrm{O}$ emissor responde com a aprovação ou negação da autorização para o credenciador que, em seguida, retorna para o estabelecimento;

e) Se aprovado, o emissor fará a liquidação da transação para a instituição financeira do credenciador, no valor total da transação descontada a taxa de intercâmbio (usualmente denominada de fee).

O cartão de crédito permitiu que as compras fossem pagas após um período sem juros, em geral, de até 45 dias. No final deste período, o consumidor pode optar por pagar o montante total em dívida, caso em que não haverá juros acumulados. Contudo, como alternativa, o consumidor pode optar por pagar apenas uma parte da dívida total, sendo a parcela restante denominada de "rotativo", saldo devedor este que passa a sofrer a incidência de juros acumulados sobre o montante utilizado, sendo todas as taxas e juros pagos para o emissor do cartão.

Tendo compreendido a etapa de entendimento do negócio, metodologicamente realiza-se avanços na seleção ou extração de dados para que posterior geração de análises prévias via Excel@ e logo a preparação da futura garimpagem de dados para estabelecimento dos clusters.

\section{CLUSTERIZAÇÃO DOS DADOS}

A identificação do perfil dos clientes inadimplentes no cartão de crédito se dá pela utilização de dados históricos dos cartões que se tornaram inadimplentes no período abrangido pela amostra e pelo ciclo de garimpagem de dados. A definição de perfil gera análise das posições mensais dos clientes em relação aos seguintes indicadores: i) Data da inadimplência e; ii) Carteira de crédito segmentada em parcelado com juros, parcelado lojista, faturados, rotativo e saque, prejuízo. Como dados de cadastro, foram considerados: 
i) Renda anual do portador; ii) Idade; ii) Atividade econômica; iii) Ocupação; iv) Sexo e; v) limite contratado.

Assim essa etapa de modelagem de dados estabeleceu uma parte inicial do processo de modelagem do data mining em que ampliou o trabalho precedentes dos mesmos autores porque incluiu além dos inadimplentes, os adimplentes que usaram o cartão de crédito. Desta forma foi possível efetivamente realizar a clusterização e suas análises via software de garimpagem de dados.

Os dados obtidos apresentados na Tabela 1 referem-se aos saldos mensais de janeiro/13 a dezembro/14 com uma amostra de 120.838 portadores pertencentes das três regionais selecionadas.

Tabela 1 - Saldos em carteira

\begin{tabular}{ll}
\hline Dados & Valor \\
\hline Total da carteira em 12/2014 & $\mathrm{R} \$ 87,2$ milhões \\
Total de portadores com cartão no período & 98.799 portadores \\
Total de portadores inadimplentes no período & 23.328 portadores \\
Carteira dos portadores inadimplentes em 12/2014 & $\mathrm{R} \$ 12,8$ milhões \\
Idade média dos portadores que se tornaram inadimplente & 37 anos \\
Idade média dos portadores adimplentes & 42 anos \\
\hline
\end{tabular}

Fonte: Dados da pesquisa

A carteira em dezembro/2014 dos cartões que se tornaram inadimplentes está composta dos seguintes saldos: Faturas a Vencer (sem juros) - R\$140 mil; Compras Parcelado Lojista (sem juros) - R\$ 165 mil; Rotativo e Saques (com juros) - R\$ 5.926 mil; Parcelado Emissor (com juros) - R\$ 92 mil; Prejuízo (baixadas como perdas) - R \$ 6.502 mil.

Para fins de sigilo bancário, os dados extraídos foram tratados no Excel@, sendo os números dos cartões e dados de identificação dos clientes totalmente omitidos. Isto, em consideração a Lei Complementar 105/2001 pela qual é dever ou obrigação das instituições financeiras de manter resguardados os dados de seus clientes.

O Gráfico 1 apresenta a evolução do total da carteira e do seu perfil no transcorrer do período da amostragem. 
Gráfico 1 - Evolução da carteira de cartões

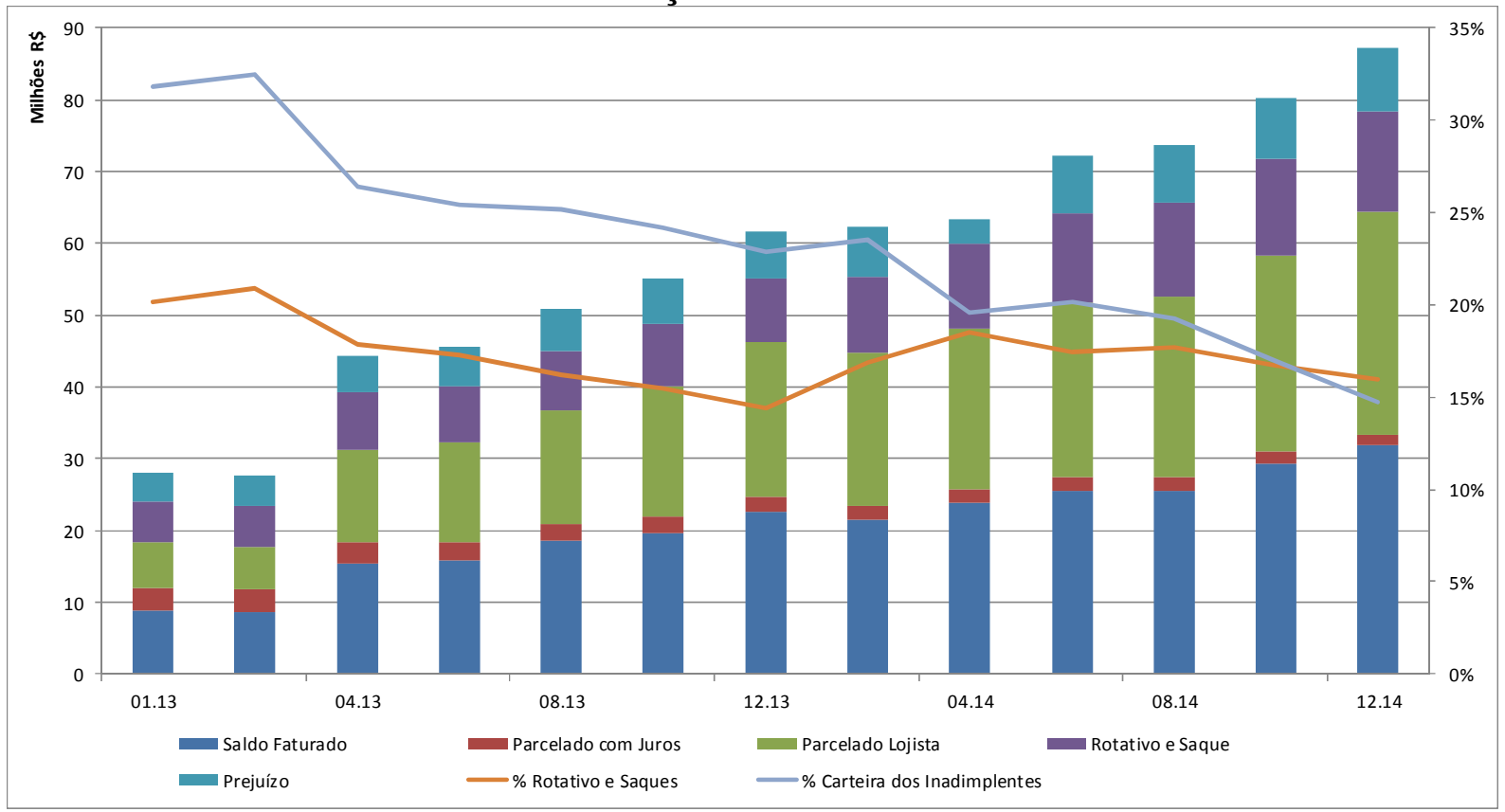

Fonte: Dados da pesquisa

Para a realização da clusterização os dados foram obtidos a partir da seguinte sequencia:

1 - Emissão dos relatórios da carteira de cartões do Sistema de Gerenciamento de Cartões (SGC);

2 - Emissão dos relatórios dos dados de cadastro do Sistema Cadastral (CCROASSO);

3 - Compilação e tabulação dos dados em Excel@;

3 - Geração dos dados em arquivo formato "arff";

4 - Importação dos dados no software Weka@;

5 - Normalização;

6 - Processamento;

7 - Geração de resultados para análise.

Dessa forma e especificamente para este trabalho, os atributos dados declarados de transferência do Excel@ para o Weka@ foram caracterizados da seguinte maneira:

@ relation Perfil

@attribute portador real

$@$ attribute regional $\{1,2,3\}$

@attribute dtinibicao real

$@$ attribute idade real

@attribute inibido $\{\mathrm{S}, \mathrm{N}\}$

$@$ attribute limite real

$@$ attribute renda real

@ attribute atividade real

$@$ attribute ocupacao real

$@$ attribute sexo $\{\mathrm{M}, \mathrm{F}\}$

@ attribute rotativo real 
@attribute parceladojuros real

@attribute faturados real

@ attribute parceladoloja real

@attribute prejuizo real

Os cinco últimos campos, com os dados da carteira, foram segregados unitariamente para cada período. Desta forma, foram declarados 5 status de carteira, multiplicados pelo período de 24 meses, perfazendo um total de 120 colunas para estes atributos. Assim, com a análise da clusterização pretende-se concluir se ocorrem padrões que permitam o agrupamento e que sejam significativos na determinação e na previsão do encaminhamento do portador para a situação de inadimplente.

\section{CONCLUSÃO}

A crescente utilização do cartão de crédito como modalidade de pagamento desperta conjuntamente a preocupação sobre possível inadimplência. Identificar o perfil da inadimplência considerando agrupamentos integrados aos perfis dos clientes é uma ação prioritária para a gestão das empresas atuantes neste setor.

Sendo assim e considerando a situação anteriormente apresentada, este trabalho ampliou posicionamento metodológico apresentado em (JANTSCH, VANTI, COSER, LAZZARI, 2014), porém aqui analisando a técnica de clusterização aliada com análise em Excel $\odot$ de agrupamento dos dados por perfil do portador. Os demais autores dão continuidade nos trabalhos, porém este especificamente detalha uma maior implementação no enfoque de mineração propriamente dita.

Como resultado desta aplicação em uma instituição financeira, aprofundou-se a etapa de entendimento do negócio concentrando-se na formação da carteira e a sua evolução até a caracterização da inadimplência. Conclui-se que dados de perfil como idade do portador, por exemplo, indicam tendência de comportamento que pode ser utilizado na gestão da carteira e como foco de ação na orientação de usuários de cartão.

Finalmente, na preparação de dados, houve a extração e a evidenciação da evolução do saldo da carteira de cartões inadimplentes e as caraterização dos grupos de adimplentes, em que a idade média é de 42 anos, superior a idade média de 37 anos dos inadimplentes. Em trabalho futuro sugere-se a implementação as etapas de Associação, Avaliação e Implantação que da mesma maneira, utilize-se software de mineração de dados e os demais dados de perfil disponíveis.

\section{REFERÊNCIAS}

AKERS, D.; GOLTER, J.; LAMM, B.; SOLT, M. Overview of recent developments in the credit card industry. FDIC Banking Review, v. 17, n. 3, p. 23-35, 2005.

BACEN a. - Banco Central do Brasil. Relatório de Estabilidade Financeira (REF) - Base de dados dos gráficos e das tabelas do capítulo 2 - Publicado em setembro/2014. Disponível em: <http://www.bcb.gov.br/htms/estabilidade/2014_09/Base_de_Dados.xls>. Acesso em 18 out 2014.

BACEN b. - Banco Central do Brasil. Relatório sobre a Indústria de Cartões de Pagamentos, $1^{a}$ edição, maio/2010. Banco Central do Brasil - Departamento de Operações 
Bancárias e de Sistema de Pagamentos. Disponível em: <http://www.bcb.gov.br/htms/spb/Relatorio_Cartoes.pdf >. Acesso em 18 out 2014.

BACEN c. - Banco Central do Brasil. Relatório de Estabilidade Financeira (REF) Setembro de 2014. Volume 13, Número 2. Disponível em: < http://www.bcb.gov.br/htms/estabilidade/2014_09/refP.pdf>. Acesso em 27 jan 2015.

BAJO, J.; BORRAJO, M. L.; DE PAZ, J. F.; CORCHADO, J. M.; PELlICER, M. A. A. multi-agent system for web-based risk management in small and medium business. Expert Systems with Applications, v. 39, n. 8, p. 6921-6931, 2012.

BENDLE, N.; HORNE, D. Visa inc. and the global payments industry. Richard Ivey School of Business Foundation, v. 2014-05-29, 2014.

BERRY, Michael J. A.; LINOFF, Gordon. Data mining techniques: for marketing, sales, and customer support. New York: John Wiley \& Sons, 1997.

COFFMAN, J. Y.; CHANDLER, G. G. Applications of performance scoring to accounts receivable management in consumer credit. Credit Research center, Krannert Graduate School of Management, Purdue University, 1983.

CRISP-DM 1.0, Step-by-step data mining guide. Pete Chapman (NCR), Julian Clinton (SPSS), Randy Kerber (NCR), Thomas Khabaza (SPSS), Thomas Reinartz (DaimlerChrysler), Colin Shearer (SPSS) and Rüdiger Wirth (DaimlerChrysler). 2000. Disponível em: <http://www.the-modeling-agency.com/crisp-dm.pdf>. Acessado em 24 out 2014.

GETTER, D. E. The credit card market: recent trends, funding, cost issues, and repricing practices. Washington D. C.: Congressional Research Service, Library of Congress, Order Code RL34393. p. 1-14, 2008.

JANTSCH, L.; COSER, T. ; LAZZARI, R.; VANTI, A. Perfil de um potencial cliente inadimplente no cartão de crédito. I congresso de controladoria e finanças. SL, 2014.

MUKHERJEE, J.; KAWDE, S. Citibank India credit cards: strategy for profitable growth. Richard Ivey School of Business Foundation, v. 5, n. 22, 2014.

OH, J. H.; JOHNSTON, W. J. Credit lender-borrower relationship in the credit card market-Implications for credit risk management strategy and relationship marketing. International Business Review, v. 23, n. 6, p. 1086-1095, 2014.

ROCHA, R.; COBO, A.; VANTI, A.; JOHANN, S. Imagens Organizacionais em Empresas Brasileiras: detecção e análise com técnicas de mineração de dados. Revista de Ciências da Administração, v.15 n.37, dez. 2013.

ROCHET, J. C.; TIROLE, J. Platform competition in two-sided markets. Journal of the European Economic Association, v. 1, n. 4, p. 990-1029, 2003. 
SANTOS, J. O.; FAMÁ, R. Avaliação da aplicabilidade de um modelo de credit scoring com variáveis sistêmicas e não-sistêmicas em carteiras de crédito bancário rotativo de pessoas físicas. Revista Contabilidade \& Finanças - USP, São Paulo, n. 44, p. 105-117, 2007.

SHARMA, S.; OSEI-BRYSON, K. M.; KASPER, G. M. Evaluation of an integrated knowledge discovery and data mining process model. Expert Systems with Applications, v. 39, n. 13, p.11335-11348, 2012.

SHEFRIN, H.; NICOLS, C. M. Credit card behavior, financial styles, and heuristics. Journal of Business Research, v. 67, n. 8, p. 1679-1687, 2014.

SHIH, C. C.; CHIANG, D.; HU, Y.; CHEN, C. C. Discovering cardholders' paymentpatterns based on clustering analysis. Expert Systems with Applications, v. 38, n. 10, p. 13284-13290, 2011.

SHMUELI, G.; KOPPIUS, O. Predictive analytics in information systems research. Management Information Systems Quarterly, v. 35, n. 3, p. 553-572, 2011.

SILVA, A. D.; XAVIER, B. M.; COSTA, H. G.; GOMES, G. R. R. Mineração de dados aplicada a relação clientes e pagamentos-estudo bibliométrico. Exatas \& Engenharia, v. 3, n. 5, p. 45-59, 2013.

TAN, P.; STEINBACH, M.; KUMAR, V. Introduction to data mining. Boston: AddisonWesley Longman Publishing, 2005.

TURBAN, E.; SHARD, R.; ARONSON, J. E.; KING, D. Business intelligence: um enfoque gerencial para a inteligência do negócio. Porto Alegre: Bookman, 2009.

WITTEN, I.; FRANK, E. Data Mining. Practical machine learning tools and techniques. New Zealand: Morgan Kaufmann Publishers, 2005.

YEH, I. C.; LIEN, C. H. The comparisons of data mining techniques for the predictive accuracy of probability of default of credit card clients. Expert Systems with Applications, v. 36, n. 2, p. 2473-2480, 2009. 